\title{
Best approximation and fixed-point theorems for discontinuous increasing maps in Banach lattices
}

\author{
Dezhou Kong ${ }^{1,2}$, Lishan Liu ${ }^{1 *}$ and Yonghong $\mathrm{Wu}^{3}$
}

\section{"Correspondence:}

mathlls@163.com

'School of Mathematical Sciences,

Qufu Normal University, Qufu,

Shandong 273165, People's

Republic of China

Full list of author information is

available at the end of the article

\begin{abstract}
In this paper, we extend and prove Ky Fan's Theorem for discontinuous increasing maps $f$ in a Banach lattice $X$ when $f$ has no compact conditions. The main tools of analysis are the variational characterization of the generalized projection operator and order-theoretic fixed-point theory. Moreover, we establish a sequence $\left\{x_{n}\right\}$ which converges strongly to the unique best approximation point. As an application of our best approximation theorems, a fixed-point theorem for non-self maps is established and proved under some conditions. Our results generalize and improve many recent results obtained by many authors.
\end{abstract}

MSC: 06F30; 47H07; 41A50; 41A65

Keywords: best approximation theorem; generalized projection operator; discontinuous increasing map; Banach lattice

\section{Introduction}

Ky Fan's approximation theorem (Theorem 2 in [1]) has attracted great attention worldwide over the last few decades. The normed space version of the theorem is as follows.

Theorem Let $K$ be a non-empty compact convex set in a normed linear space $X$. If $f$ is a continuous map from $K$ into $X$, then there exists a point $u$ in $K$ such that $\|u-f(u)\|=$ $d(f(u), K)$. The point $u$ in the theorem above is called a best approximation point off in $K$.

Ky Fan's Theorem is of great importance in nonlinear analysis, approximation theory, game theory and minimax theorems. In recent years, the theorem has been studied and generalized in various respects and applied in the analysis of many problems. Lin and Park [2], O'Regan and Shahzad [3] obtained a multivalued version of Ky Fan's result for condensing maps. Tan and Yuan [4] and Liu [5, 6] extended the theorem to the more general continuous 1-set-contractive maps under some stronger hypothesis. In the last decade, the study of random approximations and random fixed points have been a very active area of research in probabilistic functional analysis. Some results have already been achieved in this line such as those by Lin [7], Seghal and Singh [8], Seghal and Water [9], Liu [10, 11], Tan and Yuan [4], Beg and Shahzad [12]. Meanwhile, Lin [13] proved Fan's theorem for a continuous condensing map defined on a closed ball in a Banach space. Subsequently, Lin and Yen [14] proved that Ky Fan's Theorem is true for a semi-contractive

○2014 Kong et al.; licensee Springer. This is an Open Access article distributed under the terms of the Creative Commons Attribution License (http://creativecommons.org/licenses/by/2.0), which permits unrestricted use, distribution, and reproduction in any medium, provided the original work is properly cited. 
map defined on a closed convex subset of a Hilbert space. Very recently, Liu [5] proved that Ky Fan's Theorem is true for the 1-set-contractive maps defined on a bounded closed convex subset in a Banach space when $\|\cdot\|$ is replaced by Minkowski's function. For more results, the reader is referred to Shahzad [15], Markin and Shahzad [16], Amini-Harandi [17], Roux and Singh [18], Liu [19, 20], O’Regan [21], and so on.

However, so far, Ky Fan's Theorem has not been well investigated for the cases where $f$ is a discontinuous map and has no compact conditions. Partly motivated by this difficulty, Alber [22] introduced the notion of a generalized projection operator and noted that $\Pi_{C}$ can be used instead of $P_{C}$ in Banach space. Based on this concept, Li and Ok [23] proved that the metric projection operator is order-preserving in partially ordered Banach spaces. Motivated and inspired by the above mentioned work, in this paper, we obtain two best approximation theorems through the order-theoretic fixed-point theorems by using $\Pi_{C}$ instead of $P_{C}$ for reflexive, strictly convex and smooth Banach lattice. In the first best approximation theorem, we establish a sequence $\left\{x_{n}\right\}$ which converges strongly to the unique best approximation point; while in the second best approximation theorem, we obtain the existence of a minimum best approximation point and a maximum best approximation point in order intervals. As an application of our best approximation theorems, a fixedpoint theorem for non-self maps is established under some conditions which do not need to require any continuous and compact conditions on $f$.

The rest of the paper is organized as follows. In Section 2, we review the definition of the generalized projection operator in Banach spaces and its basic properties, and also give some definitions in Banach lattice and some fundamental results as preliminaries for our theorems. In Section 3, we establish the properties of the generalized projection operator in Banach lattice under some assumptions. Then we combine these results with an ordertheoretic fixed-point theorem to derive some best approximation theorems. Section 4 provides an application of these best approximation theorems to the fixed-point theory.

\section{Preliminaries}

\subsection{The generalized projection operator}

Let $X$ be a real Banach space with the dual $X^{*}$. We denote by $J$ the normalized duality mapping from $X$ to $2^{X^{*}}$ defined by

$$
J x=\left\{x^{*} \in X^{*}:\left\langle x^{*}, x\right\rangle=\left\|x^{*}\right\|\|x\|,\|x\|=\left\|x^{*}\right\|\right\},
$$

for all $x \in X$, where $\langle\cdot, \cdot\rangle$ denotes the generalized duality pairing between $X^{*}$ and $X$. It is well known that if $X$ is reflexive, strictly convex and smooth, $J$ is a surjective, injective, and single-valued map.

Let $X$ be a reflexive, strictly convex and smooth Banach space and $C$ a non-empty closed convex subset of $X$. Consider the Lyapunov functional defined by

$$
W(x, y)=\|x\|^{2}-2\langle J x, y\rangle+\|y\|^{2}, \quad \forall x, y \in X .
$$

Following Alber [22], the generalized projection operator $\Pi_{C}: X \rightarrow C$ is a map that assigns to an arbitrary point $x \in X$ the minimum point of the functional $W(x, y)$, that is, $\Pi_{C}(x)=\hat{x}$, where $\hat{x} \in C$ is the solution to the minimization problem

$$
W(x, \hat{x})=\inf _{y \in C} W(x, y) .
$$


Existence and uniqueness of the operator $\Pi_{C}$ follows from the properties of the functional $W(x, y)$ and the strict monotonicity of the mapping $J$. It is obvious from the definition of the functional $W$ that

$$
(\|x\|-\|y\|)^{2} \leq W(x, y) \leq(\|x\|+\|y\|)^{2}, \quad \forall x, y \in X .
$$

If $X$ is a Hilbert space, then $W(x, y)=\|x-y\|^{2}$ and $\Pi_{C}=P_{C}$.

If $X$ is a reflexive, strictly convex, and smooth Banach space, then for $x, y \in X, W(x, y)=0$ if and only if $x=y$. It is sufficient to show that if $W(x, y)=0$ then $x=y$. From (2.4), we have $\|x\|=\|y\|$. This implies that $\langle J x, y\rangle=\|y\|^{2}=\|J x\|^{2}$. From the definition of $J$, one has $J x=J y$. Therefore, we have $x=y$; and for more details, the reader is referred to [24, 25].

As shown in [22], the generalized projection operator on a convex closed set $C$ satisfies the following properties:

(i) The operator $\Pi_{C}$ is fixed in each point $x \in C$, i.e., $\hat{x}=x$.

(ii) The operator $\Pi_{C}$ is d-accretive in $X$, i.e.,

$$
\langle J x-J y, \hat{x}-\hat{y}\rangle \geq 0, \quad \forall x, y \in X .
$$

(iii) The point $\Pi_{C}(x)=\hat{x}$ is a generalized projection of $x$ on $C \subset X$ if and only if the following inequality is satisfied:

$$
\langle J x-J \hat{x}, \hat{x}-y\rangle \geq 0, \quad \forall y \in C .
$$

(iv) The operator $\Pi_{C}$ gives the absolutely best approximation of $x \in X$ relative to the functional $W(x, y)$, i.e.,

$$
W(\hat{x}, y)=W(x, y)-W(x, \hat{x}), \quad \forall y \in C .
$$

\subsubsection{Banach lattices}

Let $(X, \preceq)$ be a real partially ordered Banach space with the dual $X^{*}$ and $S$ be a subset of $X$. We say that an element $x$ of $X$ is an upper bound for $S$ if $S \preceq x$, that is, $y \preceq x$ for each $y \in S$ (the notation $S \succeq x$ is similarly understood). We say that $S$ is bounded from above if $S \preceq x$ for some $x \in X$, and bounded from below if $S \succeq x$ for some $x \in X$. In turn, $S$ is said to be bounded if it is bounded both from above and below. The supremum of $S$ is the minimum of the set of all upper bounds for $S$, and is denoted by $\bigvee_{X} S$ (the infimum of $S$ is denoted as $\left.\bigwedge_{X} S\right)$. As is conventional, we denote $\bigvee_{X}\{x, y\}$ as $x \vee y$, and $\bigwedge_{X}\{x, y\}$ as $x \wedge y$, for any $x, y \in X$. If $x \vee y$ and $x \wedge y$ exist for every $x$ and $y$ in $X$, we say that $(X, \preceq)$ is a lattice. And if $\bigvee_{X} S$ and $\bigwedge_{X} S$ exist for every non-empty (bounded) $S \subseteq X$, we say that $(X, \preceq)$ is a (Dedekind) complete lattice. If $Y$ is a non-empty subset of $X$ which contains $x \wedge y$ and $x \vee y$ for every $x, y \in Y$, then $Y$ is said to be a sublattice of $X$.

A normed lattice $X$ is a vector lattice with a norm $\|\cdot\|$ such that the following condition is satisfied:

$$
|x| \preceq|y| \text { implies } \quad\|x\| \leq\|y\|, \quad \text { for all } x, y \in X,
$$

where $|x|$ is defined by $|x|=x \vee(-x)$ for each $x \in X$. 
A Riesz space is a lattice $(X, \preceq)$ where $X$ is a (real) linear space whose linear structure is compatible with the partial order $\preceq$ in the sense that for all $x, y \in X, x \preceq y$ implies $\alpha x+z \preceq$ $\alpha y+z$ for every $z \in X$ and real number $\alpha>0$. The positive cone of $(X, \preceq)$ is $X_{+}=\{x \in X$ : $x \succeq \theta\}$, which is a pointed convex cone in $X$. We will assume throughout the paper that the positive cones is closed.

Let $(X, \preceq)$ be a Banach lattice, that is, $(X, \preceq)$ is an ordered Riesz space with $X$ being Banach space (if $X$ is a Hilbert space here, $(X, \preceq)$ is referred to as a Hilbert lattice). The cone $X_{+}$is said to be solid if $X_{+}$has a non-empty interior i.e. int $X_{+} \neq \emptyset$. The cone $X_{+}$is said to be normal if there is a number $K>0$ such that for all $x, y \in X, \theta \preceq x \preceq y$ implies $\|x\| \leq K\|y\|$. The least positive number satisfying this inequality is called the normal constant of $X_{+}$.

Definition 2.1 ([23]) Let $(X, \preceq)$ be a Banach lattice, a sublattice $Y$ of $X$ is said to be regular if $\|\cdot\|^{2}$ is submodular on $Y$ with respect to $\preceq$, that is,

$$
\|x \wedge y\|^{2}+\|x \vee y\|^{2} \leq\|x\|^{2}+\|y\|^{2}, \quad \forall x, y \in Y .
$$

Obviously, if $(X, \preceq)$ is itself regular, then every sublattice of $X$ is regular. We know every Hilbert normed lattice is regular and the positive cones of many Banach lattices are regular. For example, if $p \geq 2$, every sublattice $S$ of $R^{n, p}$ with $S \subseteq R_{+}^{n}$ is regular; if $p \geq 2$, every sublattice $S$ of $\ell^{p}$ with $S \subseteq \ell_{+}^{p}$ is regular.

Definition 2.2 ([23]) For any lattices $\left(X, \preceq_{X}\right)$ and $\left(Y, \preceq_{Y}\right)$, we say that a map $F: X \rightarrow Y$ is order-preserving if $x \preceq_{X} y$ implies $F(x) \preceq_{Y} F(y)$.

\subsubsection{Order-dual}

Let $(X, \preceq)$ be a Banach lattice. The dual of $\preceq$ is the partial order $\preceq^{*}$ on $X^{*}$ defined as follows:

$$
\phi \preceq^{*} \varphi \quad \text { iff } \quad\langle\varphi-\phi, x\rangle \geq 0, \quad \forall x \in X_{+} .
$$

It is well known that $\left(X^{*}, \preceq^{*}\right)$ is a Banach lattice, which is called the dual of $(X, \preceq)$. As usual, we denote the positive cone of $\left(X^{*}, \preceq^{*}\right)$ by $X_{+}^{*}$, and recall that $x \in X_{+}$iff $\langle\varphi, x\rangle \geq 0$ for every $\varphi \in X_{+}^{*}$ (see Meyer-Nieberg [26], Proposition 1.4.2).

Let $(H,\langle\cdot, \cdot\rangle)$ be a Hilbert space and $K \subseteq H$ be a closed convex cone. Recall that $K^{*}=\{x \in$ $H:\langle x, y\rangle \geq 0, \forall y \in K\}$ is called the dual cone of $K$. The cone $K$ is called subdual if $K \subseteq K^{*}$ and superdual if $K^{*} \subseteq K$. Suppose $(H,\langle\cdot, \cdot\rangle)$ be a Hilbert lattice and $K$ be its positive cone, for any $x, y \in H$, we denote the minimum (supremum) with respect to $K$ as $x \wedge y(x \vee y)$ and the minimum (supremum) with respect to $K^{*}$ as $x \wedge^{*} y\left(x \vee^{*} y\right)$.

The following fixed-point theorem is fundamental for the proof of the best approximation theorem.

Theorem 2.1 ([27]) Let $K$ be a normal and solid cone. Suppose that $f: K \rightarrow K$ is increasing and satisfies the following conditions:

(i) There exist $v \in \operatorname{int} K$ and $c>0$, such that $\theta \prec f(v) \preceq v, f(\theta) \succeq c f(v)$.

(ii) For any $0<a<b<1$ and any bounded subset $B \subset K$, there exists $\eta(a, b, B)>0$ such that

$$
f(t x) \succeq t(1+\eta) f(x), \quad \forall x \in B, t \in[a, b] .
$$


Then $f$ has a unique fixed point $x^{*}$ in $K$ such that $\theta \prec x^{*} \preceq v$. Moreover, if $\forall x_{0} \in K$ such that $x_{n}=f\left(x_{n-1}\right)(n=1,2,3, \ldots)$, then $\left\|x_{n}-x^{*}\right\| \rightarrow 0$ for $n \rightarrow \infty$.

We denote $d_{W}(x, K)=\inf \{W(x, y): y \in K\}$, where $x \in X$ and $W$ is a Lyapunov functional in $X$.

\section{Best approximation theorems}

First we establish the following properties of the generalized projection operators.

Lemma 3.1 Let $(X, \preceq)$ be a partially ordered space, and let $X_{+}$be its positive cone, then

$$
\Pi_{X_{+}}(t x)=t \Pi_{X_{+}}(x), \quad \forall t>0, x \in X .
$$

Proof For every $t>0, x \in X$, we take $y \in X_{+}$. It is obvious that $\frac{y}{t} \in X_{+}$, and so by equation (2.6) we have

$$
\left\langle J x-J \Pi_{X_{+}}(x), \Pi_{X_{+}}(x)-\frac{y}{t}\right\rangle \geq 0,
$$

and further, by the positive homogeneity of $J$, we get

$$
\left\langle J t x-J t \Pi_{X_{+}}(x), t \Pi_{X_{+}}(x)-y\right\rangle \geq 0, \quad \forall y \in X_{+} .
$$

Using (2.6), we obtain $\Pi_{X_{+}}(t x)=t \Pi_{X_{+}}(x)$.

Lemma 3.2 For a reflexive, strictly convex, and smooth Banach lattice $(X, \preceq)$, the following statements are equivalent:

$\left(\mathrm{H}_{1}\right)$ The normalized duality mapping $J$ is order-preserving;

$\left(\mathrm{H}_{2}\right) \forall x, y \in X, x \preceq y$ implies $\|J x \wedge J y\|^{2}+\|J x \vee J y\|^{2} \leq\|x\|^{2}+\|y\|^{2}$.

Proof $\left(\mathrm{H}_{1}\right) \Rightarrow\left(\mathrm{H}_{2}\right)$ If $J$ is order-preserving, for $x \preceq y$, we have $J x \wedge J y=J x, J x \vee J y=J y$. It is thus obvious that $\left(\mathrm{H}_{2}\right)$ holds.

$\left(\mathrm{H}_{2}\right) \Rightarrow\left(\mathrm{H}_{1}\right)$ Assume that $J$ is not order-preserving, then there exist $x_{0}, y_{0} \in X, x_{0} \preceq y_{0}$ such that $J x_{0} \wedge J y_{0} \neq J x_{0}, J x_{0} \vee J y_{0} \neq J y_{0}$. Since $X$ is a reflexive Banach lattice, $J$ is surjective and $X^{*}$ is a Banach lattice, which implies that there exist $z_{1} \neq x_{0}, z_{2} \neq y_{0} \in X$, such that $J x_{0} \wedge J y_{0}=J z_{1}, J x_{0} \vee J y_{0}=J z_{2}$. Indeed we have

$$
2\left\langle J x_{0} \wedge J y_{0}, x_{0}\right\rangle \leq 2\left\|J x_{0} \wedge J y_{0}\right\|\left\|x_{0}\right\| \leq\left\|J x_{0} \wedge J y_{0}\right\|^{2}+\left\|x_{0}\right\|^{2} .
$$

Since $X$ is strictly convex, which implies only in the case $z_{1}=t x_{0}, t \geq 0$, the relation $2\left\langle J x_{0} \wedge J y_{0}, x_{0}\right\rangle=2\left\|J x_{0} \wedge J y_{0}\right\|\left\|x_{0}\right\|$ holds. Moreover, only in the case $\left\|z_{1}\right\|=\left\|x_{0}\right\|$, the relation $2\left\|J x_{0} \wedge J y_{0}\right\|\left\|x_{0}\right\|=\left\|J x_{0} \wedge J y_{0}\right\|^{2}+\left\|x_{0}\right\|^{2}$ holds. This obviously implies $2\left\langle J x_{0} \wedge J y_{0}, x_{0}\right\rangle=$ $\left\|J x_{0} \wedge J y_{0}\right\|^{2}+\left\|x_{0}\right\|^{2}$ if and only if $z_{1}=x_{0}$. From the assumption, it is impossible that $z_{1}=x_{0}$. Thus

$$
2\left\langle J x_{0} \wedge J y_{0}, x_{0}\right\rangle<\left\|J x_{0} \wedge J y_{0}\right\|^{2}+\left\|x_{0}\right\|^{2} .
$$


In a similar way, we get

$$
2\left\langle J x_{0} \vee J y_{0}, y_{0}\right\rangle<\left\|J x_{0} \vee J y_{0}\right\|^{2}+\left\|y_{0}\right\|^{2} .
$$

Adding equations (3.4) and (3.5), we have

$$
\begin{aligned}
& 2\left\langle J x_{0} \wedge J y_{0}, x_{0}\right\rangle+2\left\langle J x_{0} \vee J y_{0}, y_{0}\right\rangle \\
& \quad<\left\|J x_{0} \wedge J y_{0}\right\|^{2}+\left\|x_{0}\right\|^{2}+\left\|J x_{0} \vee J y_{0}\right\|^{2}+\left\|y_{0}\right\|^{2}
\end{aligned}
$$

and

$$
\begin{aligned}
& 2\left\langle J x_{0} \wedge J y_{0}-J x_{0}, x_{0}\right\rangle+2\left\langle J x_{0} \vee J y_{0}-J y_{0}, y_{0}\right\rangle \\
& \quad<\left\|J x_{0} \wedge J y_{0}\right\|^{2}-\left\|x_{0}\right\|^{2}+\left\|J x_{0} \vee J y_{0}\right\|^{2}-\left\|y_{0}\right\|^{2} .
\end{aligned}
$$

Using $J x_{0} \wedge J y_{0}+J x_{0} \vee J y_{0}=J x_{0}+J y_{0}$, we obtain

$$
\begin{aligned}
& 2\left\langle J x_{0} \vee J y_{0}-J y_{0},-x_{0}\right\rangle+2\left\langle J x_{0} \vee J y_{0}-J y_{0}, y_{0}\right\rangle \\
& \quad<\left\|J x_{0} \wedge J y_{0}\right\|^{2}+\left\|J x_{0} \vee J y_{0}\right\|^{2}-\left\|x_{0}\right\|^{2}-\left\|y_{0}\right\|^{2},
\end{aligned}
$$

and thus

$$
2\left\langle J x_{0} \vee J y_{0}-J y_{0}, y_{0}-x_{0}\right\rangle<\left\|J x_{0} \wedge J y_{0}\right\|^{2}+\left\|J x_{0} \vee J y_{0}\right\|^{2}-\left\|x_{0}\right\|^{2}-\left\|y_{0}\right\|^{2} .
$$

Since $J x_{0} \vee J y_{0}-J y_{0} \in X_{+}^{*}$ and $y_{0}-x_{0} \in X_{+}$, we have

$$
\left\langle J x_{0} \vee J y_{0}-J y_{0}, y_{0}-x_{0}\right\rangle \geq 0 \text {. }
$$

This contradicts $\left(\mathrm{H}_{2}\right)$. So $J$ is order-preserving and the assertion is proved.

Lemma 3.3 Let $(X, \preceq)$ be a reflexive, strictly convex, smooth Banach lattice and satisfy condition $\left(\mathrm{H}_{2}\right)$ and $C$ a closed convex regular sublattice of $X$. Then, $\Pi_{C}$ is increasing.

Proof To derive a contradiction, assume that $\Pi_{C}$ is not increasing. Then, there exist $x_{0}, y_{0} \in X, x_{0} \preceq y_{0}$ such that $\Pi_{C} x_{0} \wedge \Pi_{C} y_{0} \neq \Pi_{C} x_{0}, \Pi_{C} x_{0} \vee \Pi_{C} y_{0} \neq \Pi_{C} y_{0}$. Because $C$ is a sublattice of $X$, we have $\Pi_{C} x_{0} \wedge \Pi_{C} y_{0} \in C$. It follows from the definition of $\Pi_{C}$ that $W\left(x_{0}, \Pi_{C} x_{0}\right)<W\left(x_{0}, \Pi_{C} x_{0} \wedge \Pi_{C} y_{0}\right)$, that is,

$$
2\left\langle J x_{0}, \Pi_{C} x_{0} \wedge \Pi_{C} y_{0}-\Pi_{C} x_{0}\right\rangle<\left\|\Pi_{C} x_{0} \wedge \Pi_{C} y_{0}\right\|^{2}-\left\|\Pi_{C} x_{0}\right\|^{2} .
$$

On the other hand, as $\Pi_{C} x_{0} \vee \Pi_{C} y_{0} \in C$, we trivially have $W\left(y_{0}, \Pi_{C} y_{0}\right)<W\left(y_{0}, \Pi_{C} x_{0} \vee\right.$ $\left.\Pi_{C} y_{0}\right)$, that is,

$$
2\left\langle J y_{0}, \Pi_{C} x_{0} \vee \Pi_{C} y_{0}-\Pi_{C} y_{0}\right\rangle<\left\|\Pi_{C} x_{0} \vee \Pi_{C} y_{0}\right\|^{2}-\left\|\Pi_{C} y_{0}\right\|^{2} .
$$

Using the fact that $x \vee y+x \wedge y=x+y$, we can write the inequality (3.10) as

$$
2\left\langle-J x_{0}, \Pi_{C} x_{0} \vee \Pi_{C} y_{0}-\Pi_{C} y_{0}\right\rangle<\left\|\Pi_{C} x_{0} \wedge \Pi_{C} y_{0}\right\|^{2}-\left\|\Pi_{C} x_{0}\right\|^{2} .
$$


Combining equations (3.11) and (3.12) yields

$$
\begin{aligned}
& 2\left\langle J y_{0}-J x_{0}, \Pi_{C} x_{0} \vee \Pi_{C} y_{0}-\Pi_{C} y_{0}\right\rangle \\
& \quad<\left\|\Pi_{C} x_{0} \wedge \Pi_{C} y_{0}\right\|^{2}+\left\|\Pi_{C} x_{0} \vee \Pi_{C} y_{0}\right\|^{2}-\left\|\Pi_{C} y_{0}\right\|^{2}-\left\|\Pi_{C} x_{0}\right\|^{2} .
\end{aligned}
$$

Thus, by the regularity of $C$, we get

$$
\left\langle y_{0}-J x_{0}, \Pi_{C} x_{0} \vee \Pi_{C} y_{0}-\Pi_{C} y_{0}\right\rangle<0 .
$$

By Lemma 3.2, Jy $y_{0}-J x_{0} \in X_{+}^{*}$, and so $\Pi_{C} x_{0} \vee \Pi_{C} y_{0}-\Pi_{C} y_{0}$ does not belong to $X_{+}$, which is a contradiction. This proves that $\Pi_{C}$ is increasing.

Lemma 3.4 Let $H$ be a Hilbert normed lattice with its positive cone $K$ and $C$ a closed convex sublattice of $H$. Then, $P_{C}$ is increasing.

Proof It is well known that $J$ is an identity function in Hilbert space. $\forall x, y \in K$, we have $|x-y|=(x-y) \vee(y-x) \preceq x+y$. As $H$ is a normed lattice, we get $\|x-y\| \leq\|x+y\|$, that is, $\|x-y\|^{2} \leq\|x+y\|^{2}$. Furthermore, $\langle x, y\rangle \geq 0$. Thus, $x \in K^{*}$. Conclusion: $K$ is subdual cone. Let $x, y \in H$ be such that $x \preceq y$, we have $y-x \in K \subseteq K^{*}$, that is, $x \preceq^{*} y$. Thus $x \vee^{*} y=y$ and $x \wedge^{*} y=x$. We have $\left\|x \vee^{*} y\right\|^{2}+\left\|x \wedge^{*} y\right\|^{2}=\|x\|^{2}+\|y\|^{2}$. So $\left(\mathrm{H}_{2}\right)$ holds. From Lemma 3.2.1 in [23], we know that $C$ is regular. By Lemma 3.3, $P_{C}$ is increasing.

From Theorem 2.1 and the properties of the generalized projection operator, we obtain the following best approximation theorems.

Theorem 3.1 Let $(X, \preceq)$ be a reflexive, strictly convex, smooth Banach lattice satisfying condition $\left(\mathrm{H}_{2}\right)$, and $X_{+}$be normal, solid and regular. Suppose that $f: X_{+} \rightarrow X$ is increasing and satisfies the following conditions:

$\left(\mathrm{H}_{3}\right)$ There exist $v \in \operatorname{int} X_{+}$and $c>0$, such that $f(v) \preceq v, \Pi_{X_{+}}(f(v)) \neq \theta$ and $f(\theta) \succeq c f(v)$.

$\left(\mathrm{H}_{4}\right)$ For any $0<a<b<1$ and any bounded subset $B \subset X_{+}$, there exists $\eta(a, b, B)>0$ such that

$$
f(t x) \succeq t(1+\eta) f(x), \quad \forall x \in B, t \in[a, b] .
$$

Then $f$ has a unique point $x^{*}$ in $X_{+}$, satisfying $\theta \prec x^{*} \preceq v$, such that $W\left(f\left(x^{*}\right), x^{*}\right)=$ $d_{W}\left(f\left(x^{*}\right), X_{+}\right)$. Moreover, if $x_{0} \in X_{+}$and $x_{n}=\Pi_{X_{+}}\left(f\left(x_{n-1}\right)\right)(n=1,2,3, \ldots)$, then $\left\|x_{n}-x^{*}\right\| \rightarrow$ 0 for $n \rightarrow \infty$.

Proof Define $F: X_{+} \rightarrow X_{+}$by $F(x)=\Pi_{X_{+}}(f(x))$. It is obvious that $X_{+}$is a sublattice of $X$. By Lemma 3.3, it is easy to see that $F$ is increasing. Since $\Pi_{X_{+}}$is increasing and $f(v) \preceq v$, $f(\theta) \succeq c f(v)$, we get

$$
\theta \prec \Pi_{X_{+}}(f(v)) \preceq \Pi_{X_{+}}(v)=v .
$$

Using Lemma 3.1, we have

$$
\Pi_{X_{+}}(f(\theta)) \succeq \Pi_{X_{+}}(c f(v))=c \Pi_{X_{+}}(f(v)) .
$$


From $f(t x) \succeq t(1+\eta) f(x), \forall x \in B, t \in[a, b]$, we obtain

$$
\Pi_{X_{+}}(f(t x)) \succeq \Pi_{X_{+}}(t(1+\eta) f(x))=t(1+\eta) \Pi_{X_{+}}(f(x)) .
$$

Thus $F$ satisfies all conditions of Theorem 2.1, and so $F$ has a unique fixed point $x^{*}$ in $X_{+}$, such that $\theta \prec x^{*} \preceq v$, and $\left\|x_{n}-x^{*}\right\| \rightarrow 0$ for $n \rightarrow \infty$. Now we consider $F\left(x^{*}\right)=x^{*}$, i.e. $\Pi_{X_{+}}\left(f\left(x^{*}\right)\right)=x^{*}$. By the definition of $\Pi_{X_{+}}$, we get

$$
W\left(f\left(x^{*}\right), x^{*}\right)=\inf _{y \in X_{+}} W\left(f\left(x^{*}\right), y\right)=d_{W}\left(f\left(x^{*}\right), X_{+}\right) .
$$

The assertion is proved.

Remark 3.1 In Theorem 3.1, $f$ is a discontinuous map and has no compact conditions.

Corollary 3.1 Let $H$ be a Hilbert normed lattice and its positive cone $K$ be solid. Suppose that $f: K \rightarrow H$ is increasing and satisfies $\left(\mathrm{H}_{3}\right),\left(\mathrm{H}_{4}\right)$ in $K$. Then $f$ has a unique point $x^{*}$ in $K$, satisfying $\theta \prec x^{*} \preceq v$, such that $\left\|f\left(x^{*}\right)-x^{*}\right\|=d\left(f\left(x^{*}\right), K\right)=\inf _{y \in K}\left\|f\left(x^{*}\right)-y\right\|$. Moreover, if $x_{0} \in K$ and $x_{n}=P_{K}\left(f\left(x_{n-1}\right)\right)(n=1,2,3, \ldots)$, then $\left\|x_{n}-x^{*}\right\| \rightarrow 0$ for $n \rightarrow \infty$.

Proof The assertion follows from the above Lemma 3.4 and Theorem 3.1.

Let $(X, \preceq)$ be a Banach lattice. Given $u_{0}, v_{0} \in X$ such that $u_{0} \prec v_{0}$, we denote by $\left[u_{0}, v_{0}\right]$ the set:

$$
\left[u_{0}, v_{0}\right]=\left\{z \in X: u_{0} \preceq z \preceq v_{0}\right\} .
$$

It is easy to see that $\left[u_{0}, v_{0}\right]$ is a sublattice of $X$.

Theorem 3.2 Let $(X, \preceq)$ be a reflexive, strictly convex, smooth Banach and Dedekind complete lattice satisfying condition $\left(\mathrm{H}_{2}\right)$. Suppose that $\left[u_{0}, v_{0}\right]$ is regular and $f:\left[u_{0}, v_{0}\right] \rightarrow X$ is increasing. Then, $f$ has a minimum best approximation point $x_{*}$ and a maximum best approximation point $x^{*}$ with respect to $W(x, y)$ in $\left[u_{0}, v_{0}\right]$, such that

$$
u_{0} \preceq u_{1} \preceq \cdots \preceq u_{n} \preceq \cdots \preceq x_{*} \preceq x^{*} \preceq \cdots \preceq v_{n} \preceq \cdots \preceq v_{1} \preceq v_{0},
$$

where $u_{n}=\Pi_{\left[u_{0}, v_{0}\right]}\left(f\left(u_{n-1}\right)\right), v_{n}=\Pi_{\left[u_{0}, v_{0}\right]}\left(f\left(v_{n-1}\right)\right)(n=1,2,3, \ldots)$.

Proof Define $F:\left[u_{0}, v_{0}\right] \rightarrow\left[u_{0}, v_{0}\right]$ by $F(x)=\Pi_{\left[u_{0}, v_{0}\right]}(f(x))$. From Lemma 3.3, we see that $F$ is increasing. It is easy to see that $u_{0} \preceq F\left(u_{0}\right)$ and $F\left(v_{0}\right) \preceq v_{0}$. Thus, $F$ satisfies all conditions of Theorem 2.1.2 in [28]. Then, $F$ has a minimum fixed point $x_{*}$ and a maximum fixed point $x^{*}$ and satisfies (3.18). By the definition of $\Pi_{\left[u_{0}, v_{0}\right]}$, the assertion is proved.

Remark 3.2 In Theorem 3.2, $f$ is a discontinuous map and has no compact conditions.

Example 3.1 Let $(X, \preceq)=\left(\ell^{2}, \preceq\right)$. Here $\preceq$ stands for the coordinatewise ordering. Given $u_{0}, v_{0} \in \ell^{2}$ such that $u_{0} \prec v_{0}$. Then, by Theorem 3.2, every increasing $f:\left[u_{0}, v_{0}\right] \rightarrow \ell^{2}$ has a minimum best approximation point and a maximum best approximation point with respect to $W(x, y)$ in $\left[u_{0}, v_{0}\right]$. 
Example 3.2 Let $(X, \preceq)=\left(L^{2}(\Omega), \preceq\right)$, the space of measurable functions which are 2nd power summable on $\Omega$. Endow $L^{2}(\Omega)$ with the following norm and $\preceq$ :

$$
\begin{aligned}
& \|x\|=\left(\int_{\Omega}|x(t)|^{2} d \mu\right)^{\frac{1}{2}}, \\
& L^{2}(\Omega)_{+}=\left\{x \in L^{2}(\Omega): x(t) \geq 0, \forall \text { a.e. } t \in \Omega\right\} .
\end{aligned}
$$

It is easy to see that $\left(L^{2}(\Omega), \preceq\right)$ is a Hilbert normed lattice. Given $u_{0}, v_{0} \in L^{2}(\Omega)$ such that $u_{0} \prec v_{0}$; then, by Theorem 3.2, every increasing $f:\left[u_{0}, v_{0}\right] \rightarrow L^{2}(\Omega)$ has a minimum best approximation point and a maximum best approximation point with respect to $W(x, y)$ in $\left[u_{0}, v_{0}\right]$

\section{Fixed-point theorems}

From the above best approximation theorems, we can obtain the following fixed-point theorems.

Theorem 4.1 Suppose that all conditions in Theorem 3.1 are satisfied. Moreover, one of the following conditions holds:

$\left(\mathrm{H}_{5}\right) f(\theta) \succeq \theta$;

$\left(\mathrm{H}_{6}\right) f(v) \succ \theta$.

Then, $f$ has a unique fixed point $x^{*}$ in $X_{+}$, which satisfies $\theta \prec x^{*} \preceq v$. Moreover, if $x_{0} \in X_{+}$ and $x_{n}=\Pi_{X_{+}}\left(f\left(x_{n-1}\right)\right)(n=1,2,3, \ldots)$, then $\left\|x_{n}-x^{*}\right\| \rightarrow 0$ for $n \rightarrow \infty$.

Proof It suffices to show that $x^{*}$ is the fixed point of $f$. Indeed, if $\left(\mathrm{H}_{5}\right)$ holds, using $\theta \prec$ $x^{*} \preceq v$, we get $f(\theta) \preceq f\left(x^{*}\right) \preceq f(v)$. Thus $f\left(x^{*}\right)=\left(f\left(x^{*}\right)-f(\theta)\right)+f(\theta) \in X_{+}$and $W\left(f\left(x^{*}\right), x^{*}\right)=$ $d_{W}\left(f\left(x^{*}\right), X_{+}\right)=0$. Hence $f\left(x^{*}\right)=x^{*}$.

If $\left(\mathrm{H}_{6}\right)$ holds, using $f(\theta) \succeq c f(v)$, we get $f(\theta) \in X_{+}$. From $\left(\mathrm{H}_{5}\right)$, we have $f\left(x^{*}\right)=x^{*}$. The assertion is proved.

If $f:\left[u_{0}, v_{0}\right] \rightarrow\left[u_{0}, v_{0}\right]$ is self-projective, then $\Pi_{\left[u_{0}, v_{0}\right]}(f(x))=f(x)$, and Theorem 3.2 reduces to the following fixed-point theorem:

Corollary 4.1 Let $(X, \preceq)$ be a Dedekind complete lattice. Suppose that $f:\left[u_{0}, v_{0}\right] \rightarrow X$ is increasing and satisfies:

$$
u_{0} \preceq f\left(u_{0}\right), \quad f\left(v_{0}\right) \preceq v_{0} .
$$

Then, $f$ has a minimum fixed point $x_{*}$ and a maximum fixed point $x^{*}$ in $\left[u_{0}, v_{0}\right]$. Moreover, if $u_{n}=f\left(u_{n-1}\right)$ and $v_{n}=f\left(v_{n-1}\right)(n=1,2,3, \ldots)$, then equation (3.18) holds. 


\section{Author details}

${ }^{1}$ School of Mathematical Sciences, Qufu Normal University, Qufu, Shandong 273165, People's Republic of China. ${ }^{2}$ College of Information Sciences and Engineering, Shandong Agricultural University, Taian, Shandong 271018, People's Republic of China. ${ }^{3}$ Department of Mathematics and Statistics, Curtin University of Technology, Perth, 6845, Australia.

\section{Acknowledgements}

The first and second authors were supported financially by the National Natural Science Foundation of China (11371221, 11071141), the Specialized Research Foundation for the Doctoral Program of Higher Education of China

(20123705110001) and the Program for Scientific Research Innovation Team in Colleges and Universities of Shandong Province. The third author was supported financially by the Australia Research Council through an ARC Discovery Project Grant.

Received: 30 October 2013 Accepted: 26 December 2013 Published: 22 Jan 2014

\section{References}

1. Fan, K: Extensions of two fixed point theorems of F. E. Browder. Math. Z. 112, 234-240 (1969)

2. Lin, TC, Park, S: Approximation and fixed point theorems for condensing composites of multifunctions. J. Math. Anal. Appl. 233, 1-8 (1998)

3. O'Regan, D, Shahzad, N: Approximation and fixed point theorems for countable condensing composite maps. Bull. Aust. Math. Soc. 68, 161-168 (2003)

4. Tan, KK, Yuan, XZ: Random fixed-point theorems and approximation in cones. J. Math. Anal. Appl. 85, 378-390 (1994)

5. Liu, LS: Approximation theorems and fixed point theorems for various classes of 1-set-contractive mappings in Banach spaces. Acta Math. Sin. Engl. Ser. 17, 103-112 (2001)

6. Liu, LS: Random approximations and random fixed point theorems for random 1-set-contractive non-self-maps in abstract cones. Stoch. Anal. Appl. 18, 125-144 (2000)

7. Lin, TC: Random approximations and random fixed point theorems for non-self maps. Proc. Am. Math. Soc. 103, 1129-1135 (1988)

8. Sehgal, VM, Singh, SP: On random approximations and a random fixed point theorem for set-valued mappings. Proc. Am. Math. Soc. 95, 91-94 (1985)

9. Sehgal, VM, Waters, C: Some random fixed point theorems for condensing operators. Proc. Am. Math. Soc. 90 425-429 (1984)

10. Liu, LS: Some random approximations and random fixed point theorems for 1-set-contractive random operators. Proc. Am. Math. Soc. 125, 515-521 (1997)

11. Liu, LS: Random approximations and random fixed point theorems for random 1-set-contractive non-self-maps in infinite dimensional Banach spaces. Indian J. Pure Appl. Math. 28(2), 139-150 (1997)

12. Beg, I, Shahzad, N: Random fixed points of random multivalued operators on Polish spaces. Nonlinear Anal. 20, 835-847 (1993)

13. Lin, TC: A note on a theorem of Ky Fan. Can. Math. Bull. 22, 513-515 (1979)

14. Lin, TC, Yen, CL: Applications of the proximity map to fixed point theorems in Hilbert space. J. Approx. Theory 52 , 141-148 (1988)

15. Shahzad, N: Fixed point and approximation results for multimaps in S-KKM class. Nonlinear Anal. 56, 905-918 (2004)

16. Markin, J, Shahzad, N: Best approximation theorems for nonexpansive and condensing mappings in hyperconvex spaces. Nonlinear Anal. 70, 2435-2441 (2009)

17. Amini-Harandi, A: Best and coupled best approximation theorems in abstract convex metric spaces. Nonlinear Anal. 74, 922-926 (2011)

18. Roux, D, Singh, SP: On some fixed point theorems. Int. J. Math. Math. Sci. 12, 61-64 (1989)

19. Liu, LS: On approximation theorems and fixed point theorems for non-self-mapping in infinite dimensional Banach spaces. J. Math. Anal. Appl. 188(2), 541-551 (1994)

20. Liu, LS, Li, XK: On approximation theorems and fixed point theorems for non-self-mappings in uniformly convex Banach spaces. Banyan Math. J. 4, 11-20 (1997)

21. O'Regan, D: Existence and approximation of fixed points for multivalued maps. Appl. Math. Lett. 12, 37-43 (1999)

22. Alber, Yl: Metric and generalized projection operators in Banach spaces: properties and applications. In: Kartsatos, AG (ed.) Theory and Applications of Nonlinear Operators of Accretive and Monotone Type, pp. 15-50. Dekker, New York (1996)

23. Li, J, Ok, EA: Optimal solutions to variational inequalities on Banach lattices. J. Math. Anal. Appl. 388, 1157-1165 (2012)

24. Cioranescu, I: Geometry of Banach spaces. In: Duality Mappings and Nonlinear Problems. Kluwer Academic, Dordrecht (1990)

25. Takahashi, W: Nonlinear Functional Analysis. Fixed Point Theory and Its Applications. Yokohama Publishers, Yokohama (2000)

26. Meyer-Nieberg, P: Banach Lattices. Universitext. Springer, Berlin (1991)

27. Guo, D: Existence and uniqueness of positive fixed points for mixed monotone operators and applications. Appl. Anal. 46, 91-100 (1992)

28. Guo, D, Lakshmikantham, V: Nonlinear Problems in Abstract Cones. Academic Press, New York (1988)

10.1186/1687-1812-2014-18

Cite this article as: Kong et al.: Best approximation and fixed-point theorems for discontinuous increasing maps in Banach lattices. Fixed Point Theory and Applications 2014, 2014:18 